Article

\title{
Feasibility Study of a Socially Assistive Humanoid Robot for Guiding Elderly Individuals during Walking
}

\author{
Chiara Piezzo* and Kenji Suzuki
}

Deptartment of Intelligent Interaction Technologies, University of Tsukuba, 305-8571 Ibaraki Prefecture, Japan; kenji@ieee.org

* Correspondence: chiara@ai.iit.tsukuba.ac.jp; Tel:+81-29-853-5679

Academic Editor: Dino Giuli

Received: 1 June 2017 ; Accepted: 5 July 2017 ; Published: 8 July 2017

\begin{abstract}
The impact of the world-wide ageing population has commenced with respect to society in developed countries. Several researchers focused on exploring new methods to improve the quality of life of elderly individuals by allowing them to remain independent and healthy to the maximum possible extent. For example, new walking aids are designed to allow elderly individuals to remain mobile in a safe manner because the importance of walking is well-known. The aim of the present study involves designing a humanoid robot guide as a walking trainer for elderly individuals. It is hypothesized that the same service robot provides an assistive and social contribution with respect to interaction between elderly users by motivating them to walk more and simultaneously provides assistance, such as physical assistance and gait monitoring, while walking. This study includes a detailed statement of the research problem as well as a literature review of existing studies related to walking companion robots. A user-centred design approach is adopted to report the results of the current first feasibility study by using a commercially available humanoid robot known as Pepper developed by Softbank-Aldebaran. A quantitative questionnaire was used to investigate all elements that assess intrinsic motivation in users while performing a given activity. Conversely, basic gait data were acquired through a video analysis to test the capability of the robot to modify the gait of human users. The results in terms of the feedback received from elderly subjects and the literature review improve the design of the walking trainer for elderly individuals.
\end{abstract}

Keywords: socially assistive robotics; elderly; gait; intrinsic motivation; user-centred design of robots

\section{Introduction}

\subsection{Elderly Care: New Challenges Ahead and the Importance of Walking}

There is a constant increase in the number of individuals over the age of 65 . Projections indicate this will approximately correspond to 1.5 billion in 2050 [1]. Consequently, pressure on national pension plans and long-term health care is increasing. Simultaneously, the number of working-age adults available to support elderly individuals is decreasing due to declining birth rates and increased longevity [1]. Health systems of individual governments should be prepared to address the fore-mentioned issues at the earliest.

Unesco's estimates [2] indicate that the most common diseases that affect older women over 60 years correspond to unipolar depressive disorders, hearing loss, back and neck pain, Alzheimer's disease and other dementias, and osteoarthritis. Among older men, the main causes of disability include hearing loss, back and neck pain, falls, chronic obstructive pulmonary disease, and diabetes mellitus [2].

Dementia is an umbrella term that indicates all diseases that are characterized by the progressive impairment of brain functions. Unfortunately, there are no cures for dementia to date. In order to 
decelerate or even prevent cognitive decline, doctors recommend living a healthy life by following a healthy diet, performing physical exercise, and challenging the brain via social engagement or intellectual stimulations. Thus, it is important to perform any activity that keeps the brain active and improves blood circulation. Specifically, physical exercise is an important part of a healthy lifestyle that increases the sense of wellbeing of an individual. Walking itself is a completely accessible way of physical exercise because it suits all abilities, it is free, it does not require equipment, and it can be performed anywhere. Moreover, walking in an outside environment leads to interaction between an individual and others, and thus it can correspond to a social activity that can improve an individual's mood. There is no definitive evidence from randomised trials although studies continue to explore the potential of walking as a preventive treatment for dementia as well as a disease-modifying treatment [3,4]. A main question focuses on whether physical activity directly protects an individual from dementia by providing better oxygen supply or instead whether it offers indirect protection by reducing risks, such as hypertension, that impair cognition. In any case, walking must be considered as extremely important for elderly individuals due to its role in modifying the progress of the previously mentioned diseases e.g., diabetes [5], was scientifically proven by extant studies. Moreover, monitoring the right gait parameters during walking (Section 2.3) could aid in measuring balance, and thus prevent falls that are another cause of disability based on Unesco's estimates.

\subsection{Robots for the Elderly and Acceptance Issue}

The expected growth in the population of elderly individuals has influenced researchers to design innovative solutions in the field of eldercare including robots. Assistive Human-Robot Interaction involves robots that are designed to aid individuals through physical, social, or cognitive assistance [6]. Specifically, Socially Assistive Robots are designed to help and address social needs through interactions of a specific category of users, e.g., autistic children or elderly individuals. A very famous example in the final category corresponds to Paro, namely a touch-sensitive seal robot that produces positive effects for dementia patients, such as mood improvement and a calming effect, in several experiments [7].

In the field of eldercare, a newer technology trend involves aiding elderly individuals to remain at home and live independently to the maximum possible extent. Several projects focus on ambient assisted living [8] and follow the same basic approach wherein a house becomes a smart environment that is full of sensors that provide services such as indoor user localisation, activity and event recognition, user health status assessment, etc. and a service robot is integrated with other types of assistive devices by a cloud [9]. Such robot is an embodied interface of the smart systems for net-living environment. By considering human factors, this robot aims to help people at home or in a nursing home and to contribute to enhancement of quality of life.

The robot in these cases typically corresponds to a wheel based mobile platform with a touch screen for a user interface-e.g., Kompai in the Domeo project [10], Care-O-bot in the Accompany Project [11] and ScitosG3 in the Companionable Project [12]—or with a video for telepresence—e.g., the Giraff robot in the Excite Project [13]. The shape of the service robot is related to its tasks, e.g., to provide telepresence services to enable communication with elderly individuals or with medical staff to perform basic actions such as handling an object or to provide reminders about appointments. Cloud technology is an important solution that must be considered because it makes a robot cheaper and more efficient and provides opportunities to store additional information and to access knowledge provided by other sensors around the house.

In a manner similar to any commercial product, the success of a robot mainly depends on the level of acceptability perceived by the users. In the case in the present study, target users correspond to elderly individuals who may be more vulnerable and definitely possess individual necessities that should be addressed. An individual accepts and uses a certain tool if the following requirements are met: the individual possesses a motivation to use it, he/she considers it easy to use, and he/she feels physically and psychologically comfortable using the same. Therefore, it is important to 
initially understand the motivations of elderly individuals to accept or reject a new technology. Questionnaires are usually used to quantitatively evaluate users' response during experiments. For example, the Almere Model [14] was specifically designed to test the acceptance of socially assistive robots by older adults in a care home and was then successfully used in [15].

\subsection{Research Questions and Hypotheses}

Given the reasons specified in the previous section, the final aim of the current study involves designing a robot that works as a walking trainer for elderly individuals. Training refers to the double task of motivating a user to walk more and to assisting him/her. The motivational task is designed based on intrinsic motivation theories (Section 2.1), and it should ensure that a user understands the importance of walking. Conversely, an assistive task consists of monitoring the user's gait (Section 2.3) and offering a type of physical support even through light touch contact. An appropriate design allows the same robot to make two contributions to the interaction, namely social and gait assistive contributions (Figure 1).

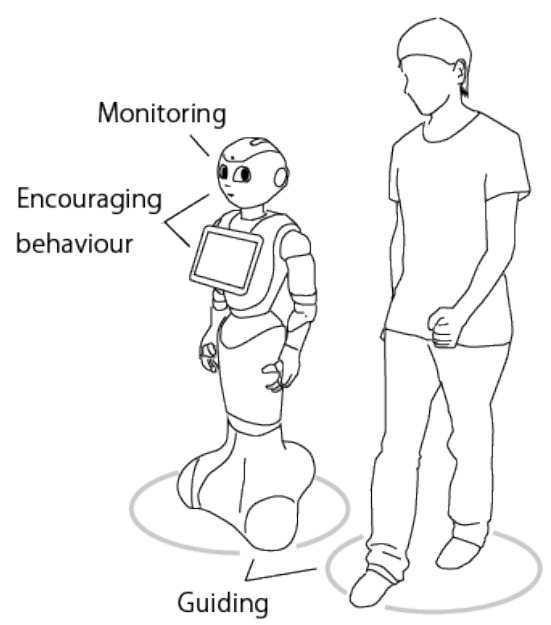

Figure 1. Potential walking method with a socially assistive humanoid robot. The picture highlights different aspects of the interaction: the same robot guides a user by encouraging him/her to walk more by using its voice and its tablet and simultaneously monitors his/her gait.

The target users correspond to older individuals who can walk by themselves or by using a walking aid, e.g., a cane or a walker and are sufficiently mentally healthy to use a robot. The specific scenario corresponds to the development of a personal robot that can stay at home with a user and convince him/her to go out given the advantages of leaving the house and meeting other individuals outside as described in Section 1.1. An initial step involves performing experiments in a care facility for elderly individuals because it includes caregivers who are familiar with the needs of their older patients, and thus useful feedback can be obtained. Additionally, a robot in a nursing home or a hospital is an attractive option because it brings a sense of novelty and enjoyment to the fixed routines of these facilities and works with overworked staff to aid them in completing tasks. In these places, a mobile robot encourages patients to walk for health benefits or to go to common areas to talk to other individuals.

A hypothesis with respect to the design is as follows: in the past, physically embodied social robots were designed to exhibit human-like social behaviours to create an interaction as similar as possible to an interaction between two individuals in which a human user knows the rules and knows how to behave. Thus, a human-shaped robot can perform human-like actions, and therefore its gestures can be easily understood and it is accepted if an appropriate behaviour is selected. Several studies indicated that physically embodied robots offer more engaging, enjoyable, and effective social interactions when compared to those of virtual agents [16]. 
A proper design is definitely required to fulfil a double task of motivating the user to walk more and assisting him as well as being accepted by elderly individuals. An understanding of the needs and expectations of target users' in a specific situation involving walking with a robot improves the design of the robot. A user-centred design approach involves including target users at the beginning of the design process and maximises the acceptability of the system [17]. The robot corresponds to an application in a real-world setting, and thus it is necessary to first understand its setting and its future users. A user-centred approach aids in designing a more natural and engaging human-robot interaction. Therefore, the first step in the current study involved performing a first feasibility study by using a commercially available robot. At this starting point, the main research questions were as follows:

- To observe the reactions of elderly individuals, i.e., if and how they accept a humanoid robot that asks them to walk together;

- To test the effectiveness of the experimental procedure and the instruments selected for evaluation;

- To investigate the strengths and the weaknesses of the current robot, its feasibility as a safe and entertaining walking trainer, and to consider improvements based on users' preferences.

These results are used in the design of the walking trainer robot.

\section{Related Works}

\subsection{Persuasive Technology}

Persuasion, or motivation is a method to increase the will to perform a certain behaviour. Psychologists distinguish between extrinsic and intrinsic motivations [18]. The first one occurs when individuals are motivated to engage in an activity to earn an external reward or avoid punishment. In contrast, the latter one involves performing an activity because it is personally rewarding, i.e., given that users find the activity itself enjoyable.

In the case of physical exercises, extant studies indicate that intrinsic motivation corresponds to most effective motivations [19]. Health professionals can better motivate older patients if they clearly explain the instructions and emphasize the importance of exercises. Reinforcement techniques are also suggested, e.g., giving positive feedback if the patient succeeds and encouragement if he/she stops, or to showing the current score compared to the point when the goal is reached.

In the early 2000s, Fogg [20] invented a new discipline of persuasive technology to explain the power of technology to change user attitudes and behaviours. Schneider et al. [21] showed the results of a long-term experiment on indoor cycling training with healthy subjects and in two different conditions: in a case they were instructed by a humanoid robot, and they were instructed by a display in another case. To the best of the authors' knowledge, Fasola et al. [22] performed the only experiment with a robot that was used to motivate older people during physical exercises. An intrinsic motivational strategy was applied following suggestions by psychologists in which a robot provided continuous feedback in real time, e.g., praise in the case of success or corrections if needed, and encouragement.

\subsection{Accompanying Robots and Proxemics}

Recently, there is an increase in studies on robots accompanying individuals. Researchers focused on the human users' social perceptions and level of comfortableness and investigated new methods to solve motion planning and control problems of detecting and following an individual in a static or dynamic environment. Table 1 summarises a few studies and shows the different locations of the robot with respect to the human user based on different tasks and scenarios. Side-by-side walks correspond to the most investigated phenomena because they are considered as the most natural type of interaction during walking. In most cases, the robot follows the human user during the interaction. Pandey et al. [23] and Leica et al. [24] defined this type of interaction as unilateral in contrast with the interaction in which a robot guides a user to a predefined goal that is subsequently defined as bilateral. 
In the final case, the robot must guide as well as interpret the human partner's behaviour-e.g., if the person experiences walking difficulties or does not want to continue following the robot, etc.-and changes its behaviour based on the same. Physical contact is present if a direct physical interface corresponds to the object of study, i.e., the user can make the robot move by touching it or if the robot provides physical assistance. Figure 2 summarises these three cases of interaction type.

When social perceptions are investigated, proxemics rules from human-human interaction studies are typically considered [25]. Proxemics is the study of how humans use and manipulate distances between each other with respect to social and cultural norms and perceptions. Individuals use proxemics signals, such as distance, body stance, hip and shoulder orientation, head poses, and eye gaze, to communicate an interest in initiating, accepting, maintaining, terminating, or avoiding social interactions. All individuals occupy space for themselves and respect the spaces occupied by others. The social-spatial distance varies with respect to the degree of familiarity between interacting humans, by the type of activity, or by the number of interactors. For example, an increase in the distance between strangers increases the comfort. The orientation is another important element that should be considered because most individuals prefer an increased distance with respect to an individual in front of them than with respect to an individual beside them [25]. Several human-robot interaction trials were performed to investigate the effect of combinations of various factors, such as robot's appearance and behaviour, or different situations, or human users' age and gender, etc., on the relative location and the comfortable distance that an individual allows between himself and a robot $[26,27]$.

Table 1. Extant studies on the specific interaction in which a human and a robot move together.

\begin{tabular}{|c|c|c|c|c|c|c|}
\hline Author & Year & $\begin{array}{l}\text { Interaction } \\
\text { Type }\end{array}$ & $\begin{array}{l}\text { Location with } \\
\text { Respect to Human }\end{array}$ & $\begin{array}{l}\text { Robot } \\
\text { Shape }\end{array}$ & $\begin{array}{l}\text { Physical } \\
\text { Contact }\end{array}$ & Purpose \\
\hline Prassler [28] & 2002 & Following human & Beside & Robotic Wheelchair & & To follow the human nurse \\
\hline Gockley [30] & 2007 & Following human & Behind & $\begin{array}{l}\text { Mobile robotic base } \\
\text { with screen }\end{array}$ & & $\begin{array}{l}\text { To find the more human-like } \\
\text { behaviour for the robot }\end{array}$ \\
\hline Hoeller [31] & 2007 & Following human & Behind & Mobile robotic base & & $\begin{array}{l}\text { To follow a human target } \\
\text { in a dynamic environment }\end{array}$ \\
\hline Kobayashi [33] & 2012 & Following human & Beside and in front & Robotic Wheelchair & & To follow the human nurse \\
\hline Ferrer [34] & 2013 & Following human & Beside & Humanoid & & $\begin{array}{l}\text { To follow the human in } \\
\text { a dynamic environment }\end{array}$ \\
\hline Morales [35] & 2014 & Following human & Beside & Humanoid & & $\begin{array}{l}\text { To find the best side-by-side } \\
\text { behaviour toward a common goal }\end{array}$ \\
\hline Chen [38] & 2010 & Guided by human & $\begin{array}{l}\text { According to } \\
\text { user }\end{array}$ & $\begin{array}{l}\text { Mobile robotic base } \\
\text { with robotic arms }\end{array}$ & $x$ & $\begin{array}{l}\text { Direct physical interface } \\
\text { to guide the robot }\end{array}$ \\
\hline Yamamoto [39] & 2013 & Guided by human & Beside & $\begin{array}{l}\text { Human-like hand and } \\
\text { head on a mobile base }\end{array}$ & $x$ & $\begin{array}{l}\text { To study side-by-side communication } \\
\text { during hand-in-hand walk }\end{array}$ \\
\hline Dabrowski [40] & 2014 & Guided by human & Beside & Humanoid & $x$ & $\begin{array}{l}\text { Direct physical interface } \\
\text { to guide the robot }\end{array}$ \\
\hline Kochigami [41] & 2015 & Guided by human & Beside and in front & Humanoid & $x$ & $\begin{array}{l}\text { Direct physical interface } \\
\text { to guide the robot }\end{array}$ \\
\hline Leica [42] & 2017 & Guided by human & In front & Mobile robotic base & $x$ & $\begin{array}{l}\text { Direct physical interface } \\
\text { to guide the robot }\end{array}$ \\
\hline
\end{tabular}




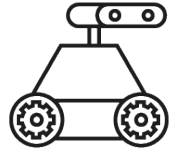

(a) Following human

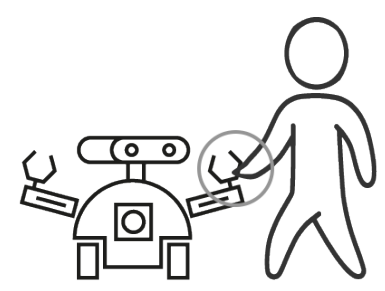

(b) Guided by human

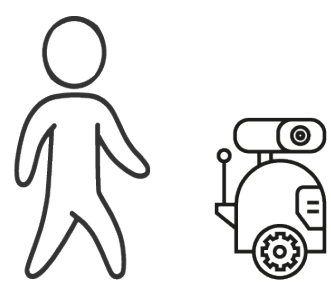

(c) Guiding human

Figure 2. (a) The robot tracks a human user and continues to follow the user (b) The human user controls and moves the robot through direct physical contact (c) The robot guides and influences the human trajectory towards a goal.

\subsection{Gait and Balance in the Elderly}

Several walking aids were designed to physically assist elderly individuals and improve their balance, e.g., walkers and canes, because balance impairment due to age is quite common. An analysis of walking patterns is considered important since gait alterations may reflect a pathology, i.e., neurological or musculoskeletal disorders [43]. With respect to quiet standing, the position or the velocity of the centre of pressure in the anterior-posterior plane is typically calculated. While walking, it is possible to measure several gait parameters in both the anterior-posterior plane and mediolateral plane including step width (the perpendicular distance from a heel/toe to the line of progression of the other heel/toe), step length (the distance from the initial contact point in a foot to the initial contact point in the other foot), cadence (walking rate, i.e., the number of steps per minute), and walking speed (the product of step length multiplied by cadence). All the fore-mentioned parameters are related to body sway [44,45], and thus observing the same can reduce the risk of falls. Falls may cause physical injuries as well as restrict mobility and lead to social isolation, which may result in depression [46].

Typically, these gait parameters are monitored in hospitals by health experts. Force plates and motion capture systems, such as VICON [47], are commonly used with good reliability. However, few observations in a hospital could not insufficient for early detection of pathologies. Furthermore, the systems require large spaces and are expensive. In the case of using markers attached to a patient's body, the placement of inaccurate markers by palpation and markers detection losses are well-known disadvantages of these systems.

Given the fore-mentioned reasons, researchers are increasingly focusing on personal robots as a better solution because the personal robot could stay at home with an elderly individual and could be provided with an inexpensive system to measure gait parameters in a non-invasive manner. Cameras are not sufficiently robust to constantly detect the legs of a user given different clothing and different light conditions. Hence, a few extant experiments successfully detected the legs of human subjects by using a laser range sensor [48,49].

\subsection{Considerations about Past Work}

In order to summarise the state of art, it is affirmed that there is an increase in studies that focus on accompanying robots during walking although difficulties persist with respect to the same, e.g., to investigate the best social behaviour for the robot and the best navigation and humans detection algorithms. To the best of the authors' knowledge, extant studies have not proposed a walking companion robot specifically designed to satisfy the needs of elderly individuals, and thus it is necessary to investigate proxemics preferences in this specific category.

This study proposes a novel concept of a walking trainer with the double task of motivating a user to walk more and to assist the user during the interaction. Extant studies proved that robots act as a companion and a motivator to perform physical exercises for elderly individuals, and thus there is a good possibility of designing an effective product that will be accepted by elderly users. In the study, 
it is proposed that a method to increase the chances of success involves including final users at the beginning of the design process.

With respect to the walking trainer robot, the most interesting challenges consist of increased research in the fields of social robots as well as assistive robots. Specifically, it is necessary to design the following:

- An effective motivational behaviour for the robot, i.e., a behaviour that can be liked and accepted by elderly individuals;

- A safe robot-human interaction model to guide human users, e.g., to a preferred location, in a specific situation in which the robot motivates an elderly individual to walk more;

- A system to observe basic gait parameters of the user to effectively monitor balance and detect anomalies and to observe the influence of the robot on the user's gait.

\section{Robotic Platform}

As specified in Section 1.3, with respect to the first feasibility study, a commercially available robot is selected because it was necessary to test the reactions of elderly individuals walking for the first time with a robot, to observe the strong points of the current robot and its limitations, to improve the future design, and to verify the effectiveness of the experimental procedures and evaluation methods.

The selected robot was named Pepper, and it was the last humanoid designed by Softbank-Aldebaran [50]. A humanoid shape is an important feature that is considered for a future walking trainer. It is expected that a humanoid shape performs better in case of interpersonal communication, i.e., verbal language and non-verbal behaviours such as proxemics and body language. Thus, a humanoid can better communicate its intentions to a human partner.

Pepper has 17 degrees of freedom. It can perform yaw and pitch rotations with its head. It can perform shoulder pitch and roll, elbow yaw and roll, and wrist yaw rotations with both arms, and it can close and open both hands. It can perform hip roll and pitch, and knee pitch rotations in the lower part of its body. The joints in the head and in the arms are sufficient to suggest the possibility of programming it to perform non-verbal gestures similar to the ones performed by humans (Section 1.3).

With respect to locomotion, Pepper possesses a system of three wheels that is definitely more stable than that with two robotic legs. Its weight approximately corresponds to $28 \mathrm{~kg}$ and its height corresponds to $1.20 \mathrm{~cm}$, and thus it is theoretically possible for an individual to walk together with the robot leaning on its shoulder in a comfortable manner (Figure 1). Extant studies indicated the efficacy of light touch [51] as well as of interpersonal touch [52] in increasing balance in several experiments and mostly in standing conditions. To the best of the authors' knowledge, the influence of interpersonal touch during gait was only studied by Zivotofsky et al. [53] simply to observe synchronisation of gait in the case of two individuals holding hands and walking together. There is a paucity of studies to guarantee the safety of elderly individuals and to effectively enhance balance.

With respect to the motivational task of our walking trainer, the design of Pepper design was potentially sufficient for the specified purpose. In addition to the humanoid shape, Pepper includes a speaker, and thus it provides positive feedback, such as encouragement and verbal praise, to increase a participant's motivation and enjoyment while walking (Section 2.1). Table 2 shows examples of the sentences spoken by Pepper during the experiment. Additionally, the robot has a tablet on the chest in which it shows the current score of the user, e.g., his velocity, the distance covered on a specific day, the number of steps needed to reach the goal fixed by the doctor for a specific day, and improvements gained since previous walks.

With respect to tracking the human user and monitoring the gait of the user, the cameras in the eyes are definitely not sufficient, and thus it was necessary to include external cameras for the sake of the experiment. A current limitation of mobile service robots corresponds to their inability to detect humans out of their sensing range. Section 1.2 specified the advantages of using a smart environment that could provide the robot with information regarding the user's position. This should be considered 
for a future design as well to integrate the walking trainer with a system that successfully monitors a user's gait parameters.

In order to perform the experiment and control the robot in an easier manner, a graphical user interface (GUI) was designed by using the TkInter package in Python [54]. In the control window, each button corresponded to a different behaviour: each behaviour was a combination of motivational utterance (Table 2) or and non-verbal gestures, e.g., head or arm rotation. The operator could move the robot by using a 3d Mouse (Space Navigator by 3dconnexion [55]) and by looking at the images captured by the robot camera and showed in the control window.

Table 2. Examples of the utterances of the robot to increase the users' motivation based on intrinsic motivational theories. During the experiment, the robot speaks in Japanese since all the participants are Japanese. The English translation is reported.

\begin{tabular}{ll}
\hline During the walk & $\begin{array}{l}\text { Try to follow me } \\
\text { You are doing great } \\
\text { You are walking really well } \\
\text { Just few meters until the end }\end{array}$ \\
\hline At the destination & $\begin{array}{l}\text { Bravo! You completed the path } \\
\text { Let's walk together again }\end{array}$ \\
\hline
\end{tabular}

\section{Experiments and Results}

\subsection{Experimental Conditions}

The experiment was conducted in two different locations, namely in a university and in a care facility in the Ibaraki prefecture where elderly individuals are allowed to stay for a maximum period of three months after hospitalisation and before becoming independent again and being able to return home. In both cases, the same experimental environment was recreated that is described in this section, and thus the experiment did not distinguish the results obtained in the two locations and a single analysis was performed.

There were a total of 8 participants (three women among them) aged between 73 to 92 years old. They were cognitively healthy, and they consistently understood and fulfilled the task expected of them. During the preparation phase, the robot and the experimental environment were introduced to the participants. The participants were clearly told that they could walk once without the robot and once with the robot (Figure 3).

In the second case, they could choose the preferred position either side-by-side by keeping the robot at their right (1) or their left (5), or behind Pepper by keeping the robot at their front-right (2), at their front (3) or at their front-left (4). Figure 4 shows the relative position of the user with respect to the robot. In all five cases, Pepper looked at an individual's eyes. The case of the robot following the user from behind was discarded because it was considered that elderly individuals must always look in front of them while walking due to safety reason. All the subjects signed the informed consent form and agreed to the video recording prior to participating in the experiment.

A Wizard of $\mathrm{Oz}$ approach was preferred due to the hardware limitations of the current robot i.e., its inability to keep tracking the human user's position and measuring the gait data in real time. In the study, the robot moves and speaks based on the actions of elderly individuals. The robot was operated in a smooth manner due to the GUI and the $3 \mathrm{~d}$ mouse. 


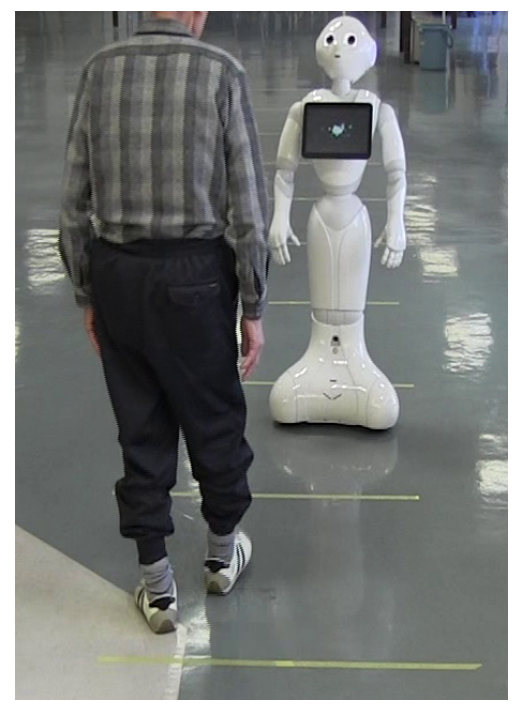

Figure 3. A male participant follows Pepper.

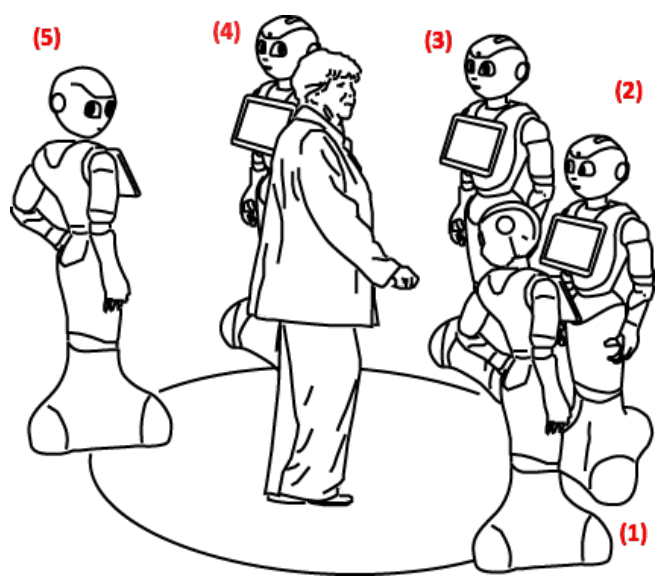

Figure 4. All the older subjects are asked as to where they prefer walking with the robot: beside the robot in (1) and (5) or behind the robot in (2), (3), (4).

\subsection{Performance Evaluation Methods for Our Walking Trainer Robot}

The actors were asked to walk straight on a PVC roll $(1 \mathrm{~mm} \times 1370 \mathrm{~mm} \times 10 \mathrm{~m})$ that was marked in advance with strips of tape at intervals of $50 \mathrm{~cm}$ along the length. The PVC roll was selected to easily calibrate videos of the camera that were subsequently analysed by using Dartfish software [56]. The software requires the camera to be perpendicular to the plane of movement and to directly face the object of interest, and thus an assistant carried the camera on a cart beside the walking path during the experiment to obtain step lengths of the participants. Consequently, the cadence and walking speed were also calculated.

With respect to the evaluation of the robot performance from a more social viewpoint, e.g., to measure the comfortableness of the users and to investigate their motivations, the Intrinsic Motivation Inventory Questionnaire (IMI) [57] that is commonly used to assess participants' level of motivation during a given activity in an experimental environment was adopted [58]. The quantitative questionnaire was administered to the participants after the end of the experiment.

The general version of the IMI questionnaire includes 7 subscales each of which corresponds to a positive or a negative predictor of intrinsic motivation according to psychologists. There are 6 positive subscales as follows: interest/enjoyment, effort/importance, perceived competence, perceived choice, value/usefulnesss, and relatedness/trust. Only 1 subscale is a negative predictor of 
intrinsic motivation, and it is related to pressure/tension. Past experiments proved the possibility of using only a few subscales without influencing the results of the others. In each subscale, there are several statements termed as items that should be rated by the participants based on a Likert scale from 1 (not at all true) to 7 (very true). Each subscale score is then calculated by averaging across all the items of the specific subscale. The full version has 45 items, and a few items in the same subscale are very similar although the use of a shorter version is considered reliable [58].

Following the instructions given by the IMI creators, a questionnaire with the following 4 subscales was obtained: enjoyment/interest $(\mathrm{E} / \mathrm{I})$, relatedness/trust $(\mathrm{R} / \mathrm{T})$, value/usefulness $(\mathrm{V} / \mathrm{U})$, and pressure/tension $(\mathrm{P} / \mathrm{T})$. For each subscale, 3 items that were randomly ordered in the questionnaire were selected. Additionally, they were modified to fit the specific activity (Table 3). The final version corresponded to a personalised questionnaire for the experiment without affecting the validity of the original. Nevertheless, Cronbach's alpha test was used to confirm the internal reliability of each subscale. With respect to the $\mathrm{E} / \mathrm{I}$, the $\mathrm{R} / \mathrm{T}$, the $\mathrm{V} / \mathrm{U}$, and the $\mathrm{P} / \mathrm{T}$ subscales, Cronbach's alpha coefficients corresponded to $0.86,0.84,0.87$, and 0.84 , respectively, and thus the modified questionnaire were considered reliable. Therefore, all the statements were well written and the participants fully comprehended the meaning of each item.

Table 3. This table shows the English version of the modified IMI questionnaire. The participants receive the Japanese version in which the items are presented in a random order and rated from 1 to 7. In the case of the items with $(\mathrm{R})$, the final score is calculated by subtracting the item response from 8.

\begin{tabular}{|c|c|c|}
\hline Subscale & $\begin{array}{c}\text { Predictor of } \\
\text { Intrinsic Motivation }\end{array}$ & Item \\
\hline Enjoyment/Interest & + & $\begin{array}{l}\text { I enjoyed walking with the robot very much } \\
\text { This walk did not hold my attention at all (R) } \\
\text { I thought this activity was quite enjoyable }\end{array}$ \\
\hline Relatedness/Trust & + & $\begin{array}{l}\text { I'd like a chance to interact with this robot more often } \\
\text { I'd really prefer not to interact with this robot in the future (R) } \\
\text { I don't feel like I could really trust this robot (R) }\end{array}$ \\
\hline Value/Usefulness & + & $\begin{array}{l}\text { I think using this robot could help me to walk more } \\
\text { I believe using this robot could be beneficial to me } \\
\text { I think this is an important activity }\end{array}$ \\
\hline Pressure/Tension & - & $\begin{array}{l}\text { I did not feel nervous at all while walking with the robot }(\mathrm{R}) \\
\text { I was very relaxed while walking }(\mathrm{R}) \\
\text { I felt pressure while walking }\end{array}$ \\
\hline
\end{tabular}

\subsection{Results}

As shown in Figure 5, all individuals did not chose to walk with Pepper side-by-side and always preferred having the robot in front of them.

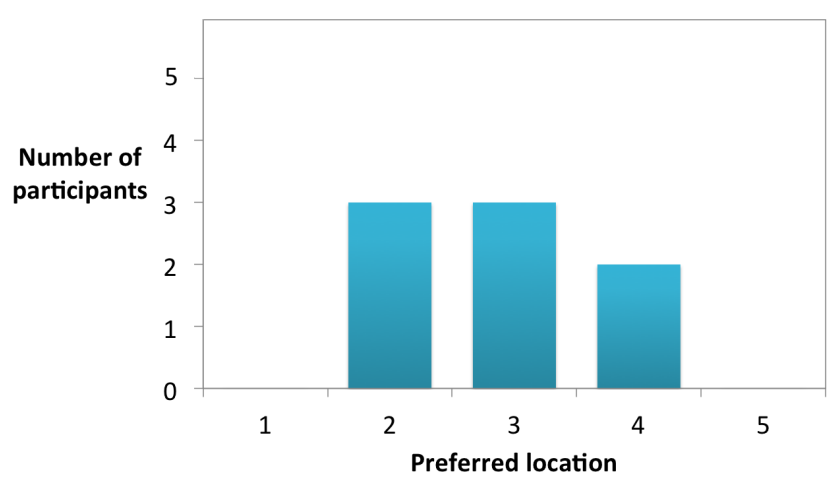

Figure 5. The figure shows the number of participants selected each specific location. The preferred location numbers correspond to the ones in Figure 4. 
Figure 6 shows the mean values and the standard deviations of the three positive subscales and of the negative subscale for all the subjects. The R/T subscale corresponds to the subscale that receives the higher score although all subscales received acceptable values. The P/T score is also acceptable.

With respect to the assistive task, Table 4 lists the walking speeds and the number of steps of each participant with respect to walking without and with Pepper.
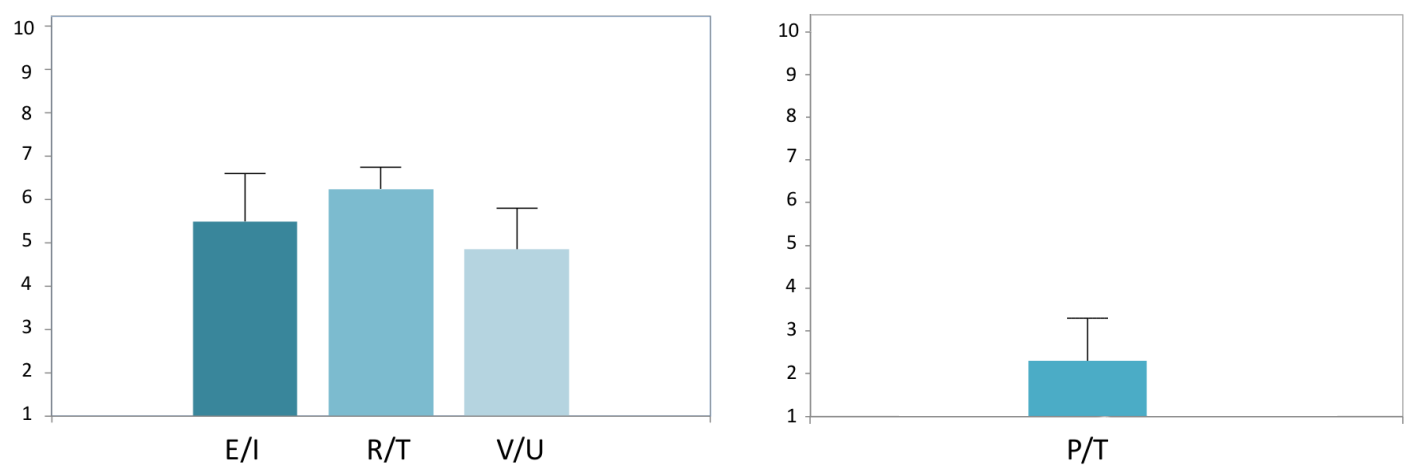

Figure 6. (a) Mean values and standard deviations of the three positive subscales in the IMI questionnaire as follows: enjoyment/interest, relatedness/trust, and value/usefulness (b) Mean value and standard deviation of the pressure/tension subscale and negative predictor of intrinsic motivation.

Table 4. This table shows the mean step length and walking speed of each participant without and with Pepper. The maximum velocity of the robot is set to a fixed value that is lower than the speed of a user $(0.25 \mathrm{~m} / \mathrm{sec})$. All the participants follow the robot by slowing down their speeds.

\begin{tabular}{ccccc}
\hline Participant & $\begin{array}{c}\text { Step Length } \mathbf{m} \\
\text { w/o Pepper }\end{array}$ & $\begin{array}{c}\text { Step Length } \mathbf{m} \\
\text { w Pepper }\end{array}$ & $\begin{array}{c}\text { Walking Speed } \mathbf{m} / \mathbf{s} \\
\text { w/o Pepper }\end{array}$ & $\begin{array}{c}\text { Walking Speed } \mathbf{~} / \mathbf{s} \\
\text { w Pepper }\end{array}$ \\
\hline 1 & 0.46 & 0.35 & 0.63 & 0.29 \\
2 & 0.43 & 0.20 & 0.50 & 0.17 \\
3 & 0.36 & 0.23 & 0.45 & 0.25 \\
4 & 0.39 & 0.23 & 0.56 & 0.26 \\
5 & 0.41 & 0.26 & 0.58 & 0.26 \\
6 & 0.47 & 0.35 & 0.65 & 0.28 \\
7 & 0.46 & 0.34 & 0.65 & 0.28 \\
8 & 0.44 & 0.21 & 0.53 & 0.22 \\
\hline
\end{tabular}

\section{Discussion}

With respect to all the elderly participants, they interacted with a humanoid robot for the first time, and thus additional care was exercised to make them feel safe and comfortable before, during, and after the experiment. It was especially to not exhaust the elderly individuals in the care facility since something new in their routines can exhaust them, and thus they were asked to walk on the experimental path just once without the robot to indicate the possibility of monitoring their walking speed in normal conditions and then to walk once with the robot in a preferred position and for a preferred distance.

With respect to the selection of preferred location as shown in Figure 5, all individuals did not select the side-by-side walk. This was considerably unexpected because it was assumed that a side-by-side walk was more natural since this is normally adopted by two individuals when they walk together. The participants appeared to view Pepper more as a guide to follow as opposed to an accompanying companion or a caregiver to walk with. Nevertheless, this corresponded to the first trial with the robot, and trust typically corresponds to something that must be earned. Pepper received a good score on the $\mathrm{R} / \mathrm{T}$ subscale although it is a score that was given after the experiment and not before. The preference for the position influences future design including the position of the 
laser range sensor at the bottom of the robot, gait monitoring, the position of a possible handlebar, and interaction behaviour.

Another misconception involved considering the first reaction of elderly individuals to the robot in which they were expected to be significantly mistrustful, fearful, and not confident of the robot's abilities. Although this was true since nobody selected the side-by-side walk, it was not completely true. For example, it was observed that some individuals did not exhibit a sense of danger and walked very close to Pepper touching it without fear while the experiment was performed., Thus, a safer robot should be considered to design a softer robot. The cute design certainly influenced the positive reaction although it was also a disadvantage since Pepper includes details that are so well designed that sometimes the participants expected a lot from the robot even with respect to tasks not implemented in this experiment such as shaking the robot's hand or holding a proper conversation.

With respect to the motivational task, good results were obtained in the IMI questionnaire in all the subscales (Figure 6). The IMI questionnaire results indicated that intrinsic motivation theory was efficient for this purpose as suggested by extant studies. Simultaneously, the Cronbach's alpha test proved the reliability of the modified questionnaire. A higher score is obtained if the interaction is personalised according to the user's preferences and characteristics. For example, there is a positive impact on the interaction if the robot recognises the human user, if it remembers the relative distance or the walking speed he prefers, or if it remembers what are his interests and what he likes talking about during the walk etc.

With respect to the gait performance as shown in Table 4, it was decided to move the robot at a slower speed when compared to the subject's normal speed to study whether or not the subject could follow and adapt his own speed to the robot's speed. All the subjects could execute the same, and this implied that a walking trainer robot successfully maintained or corrected the gait of an elderly individual if an appropriate motivational behaviour is selected. As a reminder, the target users include older individuals without a physical or mental pathology although they may be balance-impaired due to their age.

As a walking trainer, the robot guides the subjects, and thus it influences the subject's behaviour. Simultaneously, the robot's behaviour is influenced by the subject, e.g., if the subject stops or slows down, then the robot catches him, attempts to get his attention again, and motivates him to maintain the walking pace. In the feasibility study, the robot was controlled by a human operator, and this bilateral interaction in the future will be performed by a control algorithm that elaborates the response from sensors, e.g., the response regarding a user's position and gait data.

In this study, the feasibility of the propose concept and the reliability of our evaluation method and experimental protocol were proven, Additionally, useful comments were elicited to improve the design of a future robot. The development of first prototype will definitely require additional long-term trials with additional subjects to validate its effectiveness as a walking trainer. Currently, it is possible to investigate other conditions in addition to the relative position, e.g., different robot speeds, different distances, etc., and to thereby enhance the quality of the experiments.

\section{Conclusions}

The focus of this study involved determining key factors that influence a walking trainer robot for elderly individuals and to validate its feasibility. This concept evolved from observations of current society problems and the needs of elderly individuals. Section 1 shows the social and economic impact of the ageing population on society in developed countries and how it will worsen based on all projections. An active study aim involves improving the mobility of elderly individuals because the importance of walking for mental and physical health is scientifically established. Section 2 discusses evidence for the feasibility of the proposed concept with respect to state of art studies. It is observed in similar extant studies, and the novelty of the study in socially contributing to gait training is validated. A walking trainer for the elderly is validated as a useful tool that should at the very least include effective motivational behaviour, a safe and comfortable interaction with the user, and provide 
continuous gait analysis. A robot should be accepted by its target users to ensure success, and thus the study opted for a user-centred design and a first feasibility study was performed by using an available humanoid robot.

It was observed that the elderly individuals were not afraid of Pepper once they commenced the experiment even if it was the first time that they interacted with a humanoid, and they could successfully follow the speed of the robot. Good feedback was obtained from our older participants, and thus a future study will involve developing an independent robot prototype. With respect to the tools for the evaluation, the IMI questionnaire was proven as appropriate to determine intrinsic motivation in users. Cameras are sufficiently robust to monitor gait parameters, and thus it is planned to use a laser range sensor in a future design.

The introduction of a robot in a nursing home is a good idea, e.g., since it could help the overworked staff or bring a sense of refreshment in the facility. Nevertheless, it could be more useful to design a personal robot that can stay at home because elderly individuals typically prefer to remain independent in their homes although it is sometimes not possible due to poor health conditions. The current new trend of smart homes can help them in fulfil their wishes and keep them healthy in their own houses, and this would even result in a reduction of costs with respect to the national health services provided by individual countries. Future internet technologies open new opportunities to provide advanced robotic services since service robots would become cheaper and smarter due to the cooperation of all sensors and devices in a house. In this type of scenario, a walking trainer could obtain more data with respect to a user including location, activities, and health measurements, etc. and send the same to doctors or families and share information obtained from a cloud with elderly individuals. For example, a robot could examine if a user's friends are walking outside in a specific moment and could suggest going out and reaching out to them for a nice talk and walk together.

In this article, a novel approach was presented with respect to designing a human-robot interaction including starting to clearly define the research problem, to include final users since the beginning, and how to understand and evaluate the interaction.

Acknowledgments: We gratefully acknowledge Yoshi Iwamoto, M.D., Ph.D, the director of the nursing home, who helped finding the participants and providing the experimental space.

Author Contributions: Chiara Piezzo and Kenji Suzuki conceived and designed the experiments; Chiara Piezzo performed the experiments, and analysed the data. Chiara Piezzo wrote the paper; Kenji Suzuki supervised the whole work and provide feedback in the writing of the paper.

Conflicts of Interest: The authors declare no conflict of interest.

\section{References}

1. Bremner, J.; Frost, A.; Haub, C.; Mather, M.; Ringheim, K.; Zuehlke, E. World population highlights: Key findings from PRB's 2010 world population data sheet. Popul. Bull. 2010, 65, 2-3

2. World Population Ageing Report 2015-The United Nations. Available online: http://www.un.org/en/ development/desa/population/publications/pdf/ageing/WPA2015_Report.pdf (accessed on 1 April 2017).

3. Ahlskog, J.E.; Geda, Y.E.; Graff-Radford, N.R.; Petersen, R.C. Physical Exercise as a Preventive or Disease-Modifying Treatment of Dementia and Brain Aging. Mayo Clin. Proc. 2011, 86, 876-884, doi:10.4065/mcp.2011.0252.

4. Atherton, N.; Bridle, C.; Brown, D.; Collins, H.; Dosanjh, S.; Griffiths, F.; Lamb, S.E. Dementia and Physical Activity (DAPA) - An exercise intervention to improve cognition in people with mild to moderate dementia: Study protocol for a randomized controlled trial. Trials 2016, 17, 165, doi:10.1186/s13063-016-1288-2.

5. Di Loreto, C.; Fanelli, C.; Lucidi, P.; Murdolo, G.; de Cicco, A.; Parlanti, N.; Ranchelli, A.; Fatone, C.; Taglioni, C.; Santeusanio, F.; et al. Make your diabetic patients walk: Long-term impact of different amounts of physical activity on type 2 diabetes. Diabetes Care 2005, 28, 1295-1302, doi:10.2337/diacare.28.6.1295.

6. McColl, D.; Hong, A.; Hatakeyama, N.; Nejat, G.; Benhabib, B. A Survey of Autonomous Human Affect Detection Methods for Social Robots Engaged in Natural HRI. J. Intell. Robot. Syst. 2016, 82, 101-133, doi:10.1007/s10846-015-0259-2. 
7. PARO Therapeutic Robot. Available online: http://www.parorobots.com/ (accessed on 1 April 2017).

8. Ambient Assisted Living Joint Programme. Available online: http://www.aal-europe.eu/about/objectives/ (accessed on 1 April 2017).

9. Bonaccorsi, M.; Fiorini, L.; Cavallo, F.; Saffiotti, A.; Dario, P. A Cloud Robotics Solution to Improve Social Assistive Robots for Active and Healthy Aging. Int. J. Soc. Robot. 2016, 8, 393-408, doi:10.1007/s12369-016-0351-1.

10. Robosoft Kompai. Available online: http://www.robosoft.com/ (accessed on 1 April 2017).

11. Accompany-Acceptable robotiCs COMPanions for AgeiNg Years. Available online: http:// rehabilitationrobotics.net/cms2/ (accessed on 1 April 2017).

12. Companionable Project. Available online: http://www.companionable.net/ (accessed on 1 April 2017).

13. Giraff Technologies AB. Available online: http://www.giraff.org/ (accessed on 1 April 2017).

14. Heerink, M.; Krose, B.; Evers, V.; Wielinga, B. Assessing Acceptance of Assistive Social Agent Technology by Older Adults: The Almere Model. Int. J. Soc. Robot. 2010, 2, 361-375, doi:10.1007/s12369-010-0068-5.

15. Torta, E.; Werner, F.; Johnson, D.O.; Juola, J.F.; Cuijpers, R.H.; Bazzani, M.; Oberzaucher, J.; Lemberger, J.; Lewy, H.; Bregman, J. Evaluation of Small Socially-Assistive Humanoid Robot in Intelligent Homes for the Care of the Elderly. J. Intell. Robot. Syst. 2014, 76, 57-71, doi:10.1007/s10846-013-0019-0.

16. Bainbridge, W.A.; Hart, J.W.; Kim, E.S.; Scassellati, B. The Benefits of Interactions with Physically Present Robots over Video-Displayed Agents. Int. J. Soc. Robot. 2011, 3, 41-52, doi:10.1007/s12369-010-0082-7.

17. Doering, N.; Poeschl, S.; Gross, H.M.; Bley, A.; Martin, C.; Boehme, H.J. User-centered Design and Evaluation of a Mobile Shopping Robot. Int. J. Soc. Robot. 2015, 7, 203-225, doi:10.1007/s12369-014-0257-8.

18. Ryan, R.M.; Deci, E.L. Intrinsic and Extrinsic Motivations: Classic Definitions and New Directions. Contemp. Educ. Psychol. 2000, 25, 54-67, doi:10.1006/ceps.1999.1020.

19. Phillips, E.M.; Schneider, J.C.; Mercer, G.R. Motivating Elders to Initiate and Maintain Exercise. Arch. Phys. Med. Rehabil. 2004, 85, 52-57, doi:10.1016/j.apmr.2004.03.012.

20. Fogg, B.J. Persuasive technology: Using Computers to Change What We Think and Do, 1st ed.; Morgan Kaufmann: Boston, MA, USA, 2003, ISBN 978-1558606432.

21. Schneider, S.; Sussenbach, L.; Berger, I.; Kummert, F. Long-term Feedback Mechanisms for Robotic Assisted Indoor Cycling Training. In Proceedings of the 3rd International Conference on Human-Agent Interaction, Daegu, Kyungpook, Korea, 21-24 October 2015; pp. 157-164.

22. Fasola, J.; Mataric, M.J. Robot Exercise Instructor: A Socially Assistive Robot System to Monitor and Encourage Physical Exercise for the Elderly. In Proceedings of the International Symposium on Robot and Human Interactive Communication, Viareggio, Italy, 13-15 September 2010; pp. 416-421.

23. Pandey, A.K.; Alami, R. Towards a Sociable Robot Guide which Respects and Supports the Human Activity. In Proceeding of the International Conference on Automation Science and Engineering, Bangalore, India, 22-25 August 2009; pp. 262-267.

24. Leica, P.; Toibero, J.M.; Roberti, F.; Carelli, R. Switched Control to Robot-Human Bilateral Interaction for Guiding People. J. Intell. Robot. Syst. 2015, 77, 73-93, doi:10.1007/s10846-014-0098-6.

25. Hall, E.T. The Hidden Dimension, 27th ed.; Anchor: New York, NY, USA, 1990, ISBN 0-385-08476-5.

26. Kosinski, T.; Obaid, M.; Wozniak, P.W.; Fjeld, M.; Kucharski, J. A Fuzzy Data-based Model for Human-Robot Proxemics. In Proceedings of the International Symposium on Robot and Human Interactive Communication, New York, NY, USA, 26-31 August 2016; pp. 335-340.

27. Obaid, M.; Sandoval, E.B.; Zlotowski, J.; Moltchanova, E.; Basedow, E.M.; Bartneck, C. Stop! That is Close Enough. How Body Postures Influence Human-Robot Proximity. In Proceedings of the International Symposium on Robot and Human Interactive Communication, New York, NY, USA, 26-31 August 2016; pp. 354-361.

28. Prassler, E.; Bank, D.; Kluge, B. Key Technologies in Robot Assistants: Motion Coordination between a Human and a Mobile Robot. Trans. Control Autom. Syst. Eng. 2002, 4, 56-61.

29. Jin, T.S.; Lee, J.M.; Hashimoto, H. Position Control of Mobile Robot for Human-Following in Intelligent Space with Distributed Sensors. Int. J. Control Autom. Syst. 2006, 4, 204-216.

30. Gockley, R.; Forlizzi, J.; Simmons, R. Natural Person-Following Behaviour for Social Robots. In Proceedings of the ACM/IEEE International Conference on Human-Robot Interaction, Arlington, VA, USA, 9-11 March 2007; pp. 17-24. 
31. Hoeller, F.; Schulz, D.; Moors, M.; Schneider, F.E. Accompanying Persons with a Mobile Robot using Motion Prediction and Probabilistic Roadmaps. In Proceedings of the International Conference on Intelligent Robots and Systems, San Diego, CA, USA, 29 October-2 November 2007; pp. 1260-1265.

32. Morales, Y.; Satake, S.; Huq, R.; Kanda, T.; Hagita, N. How Do People Walk Side-By-Side?-Using A Computational Model of Human Behaviour For A Social Robot. In Proceedings of the International Conference on Human-Robot interaction, Boston, MA, USA, 5-8 March 2012; pp. 301-308.

33. Kobayashi, Y.; Kinpara, Y.; Takano, E.; Kuno, Y.; Yamazaki, K.; Yamazaki, A. Robotic Wheelchair Moving with Caregiver Collaboratively Depending on Circumstances. In Proceedings of the International Conference on Human Factors in Computing Systems, Vancouver, BC, Canada, 7-12 May 2011; pp. 2239-2244.

34. Ferrer, G.; Garrell, A.; Sanfeliu, A. Robot Companion: A Social-Force based approach with Human Awareness-Navigation in Crowded Environments, In Proceedings of the International Conference on Intelligent Robots and Systems, Tokyo, Japan, 3-7 November 2013; pp. 1688-1694.

35. Morales, Y.; Kanda, T.; Hagita, N. Walking Together: Side-by-Side Walking Model for an Interacting Robot. J. Hum. Robot Interact. 2014, 3, 50-73, doi:10.5898/JHRI.3.2.Morales

36. Oishi, S.; Kohari, Y.; Miura, J. Toward a Robotic Attendant Adaptively Behaving According to Human State. In Proceedings of the International Symposium on Robot and Human Interactive Communication, New York, NY, USA, 26-31 August 2016; pp. 1038-1043.

37. Spenko, M.; Yu, H.; Dubowsky, S. Robotic Personal Aids for Mobility and Monitoring for the Elderly. IEEE Trans. Neural Syst. Rehabil. Eng. 2006, 14, 344-351, doi:10.1109/TNSRE.2006.881534.

38. Chen, T.L.; King, C.H.A.; Thomaz, A.L.; Kemp, C.C. An Investigation of Responses to Robot-Initiated Touch in a Nursing Context. Int. J. Soc. Robot. 2014, 6, 141-161, doi:10.1007/s12369-013-0215-x.

39. Yamamoto, N.; Fukamachi, K.; Takeda, Y.; De Silva, P.R.S.; Okada, M. Mako-no-Te: To Explore Side-to-Side Communication through the Intersubjectectivity. In Proceedings of the International Conference on Human-Agent Interaction, Sapporo, Japan, 7-9 August 2013.

40. Dabrowski, J.; Kondaxakis, P.; Kyrki, V. Holding Hands? Guiding Humanoid Walking Using Sensorless Force Control. In Proceedings of the International Symposium on Robot and Human Interactive Communication, Edinburgh, Scotland, UK, 25-29 August 2014; pp. 180-185.

41. Kochigami, K.; Jiang, J.; Kakehashi, Y.; Au, C.; Kakiuchi, Y.; Okada, K.; Inaba, M. Walking Together Hand in Hand: Design and Evaluation of Autonomous Robot System that a Robot Recognizes Moving Direction with a Child's Assistance of Pulling its Hand. In Proceedings of the International Symposium on System Integration, Nagoya, Japan, 11-13 December 2015; pp. 738-743.

42. Leica, P; Roberti, F.; Monllor, M.; Toibero, J.M; Carelli, R. Control of Bidirectional Physical Human? Robot Interaction based on the Human Intention. J. Intell. Serv. Robot. 2016, 10, 31-40, doi:10.1007/s11370-016-0207-4.

43. Capezuti, L.; Malone, M.L.; Katz, P.R.; Mezey, M. The Encyclopedia of Elder Care: The Comprehensive Resource on Geriatric Health and Social Care, 3rd ed.; Springer Publishing Company: New York, NY, USA, 2013; pp. 319-324, ISBN 0826137350

44. Helbostad, J.L.; Moe-Nilssen, R. The Effect of Gait Speed on Lateral Balance Control During Walking in Healthy Elderly. Gait Posture 2003, 18, 27-36, doi:10.1016/S0966-6362(02)00197-2.

45. Owings, T.M.; Grabiner, M.D. Step Width Variability but not Step Length Variability or Step Time Variability, Discriminates Gait of Healthy Young and Older Adults During Treadmill Locomotion. J. Biomech. 2004, 37, 935-938, doi:10.1016/j.jbiomech.2003.11.012.

46. Bradley, S.M. Falls in Older Adults. Mt. Sinai J. Med. 2011, 78, 590-595, doi:10.1002/msj.20280.

47. Motion Capture Systems-VICON. Available online: http://www.vicon.com (accessed on 1 April 2017).

48. Palleja, T.; Teixido, M.; Tresanchez, M.; Palacin, J. Meauring Gait Using a Ground Laser Range Sensor. Sensors 2009, 9, 9133-9146, doi:10.3390/s91109133.

49. Yorozu, A.; Takahashi, M. Development of Gait Measurement Robot Using Laser Range Sensor for Evaluating Long-distance Walking Ability in the Elderly. In Proceedings of the International Conference on Intelligent Robots and Systems, Hamburg, Germany, 28 September-2 October 2015; pp. 4888-4893.

50. Pepper Softbank Robotics. Available online: https://www.ald.softbankrobotics.com/en/cool-robots/ pepper (accessed on 1 April 2017).

51. Jeka, J.J. Light Touch Contact as a Balance Aid. Phys. Ther. 1997, 77, 476-487. 
52. Johannsen, L.; Guzman-Garcia, A.; Wing, A.M. Interpersonal Light Touch Assists Balance in the Elderly. J. Mot. Behav. 2009, 41, 397-399, doi:10.3200/35-09-001.

53. Zivotofsky, A.Z; Hausdorff, J.M. The Sensory Feedback Mechanisms Enabling Couples to Walk Synchronously: An Initial Investigation. J. Neuroeng. Rehabil. 2007, 4, doi:10.1186/1743-0003-4-28.

54. TkInter-Python wiki. Available online: https://wiki.python.org/moin/TkInter (accessed on 1 April 2017).

55. 3Dconnexion SpaceMouse. Available online: https://www.3dconnexion.com (accessed on 1 April 2017).

56. Dartfish Software. Available online: http://www.dartfish.com (accessed on 1 April 2017).

57. Self-Determination Theory: An approach to human motivation and personality-Questionnaires, Intrinsic Motivation Inventory. Available online: http:/ / selfdeterminationtheory.org (accessed on 1 April 2017).

58. Colombo, R.; Pisano, F.; Mazzone, A.; Delconte, C.; Micera, S.; Carrozza, M.C.; Dario, P.; Minuco, P. Design Strategies to Improve Patient Motivation During Robot-aided Rehabilitation. J. Neuroeng. Rehabil. 2007, 4, doi:10.1186/1743-0003-4-3.

59. Mechling, H. Dementia and physical activity. Eur. Rev. Aging Phys. Act. 2008, 5, 1-3, doi:10.1007/s11556-008-0033-6.

(C) 2017 by the authors. Licensee MDPI, Basel, Switzerland. This article is an open access article distributed under the terms and conditions of the Creative Commons Attribution (CC BY) license (http:/ / creativecommons.org/licenses/by/4.0/). 